\title{
Extraction of spectral based measures from MEG background oscillations in Alzheimer's disease
}

Jesús Poza ${ }^{1}$, Roberto Hornero $^{1}$, Daniel Abásolo ${ }^{1}$, Alberto Fernández ${ }^{2}$, María

\author{
García $^{1}$
}

${ }^{1}$ Grupo de Ingeniería Biomédica, E.T.S. Ingenieros de Telecomunicación, University of Valladolid, Spain

${ }^{2}$ Centro de Magnetoencefalografía Dr. Pérez-Modrego, Facultad de Medicina, University Complutense of Madrid, Spain

AUTHOR'S ADDRESS: Jesús Poza

E.T.S. Ingenieros de Telecomunicación

University of Valladolid

Camino del Cementerio s/n

47011 - Valladolid (Spain)

Phone: +34983 42300, ext. 5569

Fax: +34 983423667

E-mail: jespoz@tel.uva.es 


\begin{abstract}
In this study, we explored the ability of several spectral based measures to summarize the information of the power spectral density (PSD) function from spontaneous magnetoencephalographic (MEG) activity in Alzheimer's disease (AD). The MEGs of 20 AD patients and 21 elderly controls were recorded with eyes closed at rest during 5 min from 148 channels. Five spectral parameters were estimated from PSD: mean frequency (MF), individual alpha frequency (IAF), transition frequency (TF), 95\% spectral edge frequency (SEF95) and spectral entropy (SE). To reduce the dimensionality of the problem, we applied a principal component analysis. According to our results, MF was the best discriminating index between both groups $(85.00 \%$ sensitivity, $85.71 \%$ specificity) indicating a shift to the left of the power spectrum in AD. A significant MEG slowing was also observed using both IAF ( $p$ $<0.001)$ and TF $(p<0.01)$. The lowest classification statistics $(65 \%$ sensitivity, $76.19 \%$ specificity) were obtained with SEF95. However, these results were also significant $(p<$ 0.05). This fact points out that there is a variation in the spectral content at high frequencies of AD patients and controls. Finally, a significant decrease of irregularity in the AD group was observed with SE, with results close to those obtained with MF (90.00\% sensitivity, $76.19 \%$ specificity). In conclusion, a complete description of PSD can help to increase our insight into brain dysfunction in $\mathrm{AD}$ and to extract spectral patterns specific to the disease.
\end{abstract}

Keywords: Alzheimer's disease; Magnetoencephalogram; Power Spectral Density; Principal Component Analysis. 


\section{Introduction}

Alzheimer's disease (AD) is a progressive and irreversible brain disorder of the central nervous system, which represents the most common form of dementia in western countries [1]. AD is characterized by loss of mental and physical abilities severe enough to interfere with normal activities of daily living.

Usually, AD starts to destroy neurons (nerve cells) in parts of the brain that are responsible for storing and retrieving new information, including the temporal lobe and the hippocampus. Then, $\mathrm{AD}$ affects the areas responsible for language and reasoning in the cerebral cortex. Eventually, many other areas of the brain are affected by atrophy with the result that $\mathrm{AD}$ patients may wander, be unable to engage in conversation, appear uncooperative and non-responsive, become helpless and incontinent and, in extreme cases, need complete care. The autopsy of AD patients shows that the regions of the brain affected by the disease contain two abnormal structures: neurofibrillary tangles and beta-amyloid plaques. Neurofibrillary tangles are twisted fibers caused by changes in the tau protein. The beta-amyloid plaques are formed by insoluble filamentous deposits of beta-amyloid, found in the minute gaps between nerve cells. Although it is not exactly clear how these structures cause problems, many researchers believe that their formation blocks the normal transport of the electrical messages between the neurons and, finally, leads to neuronal death [2].

Nowadays, there is no single test that can accurately predict who will develop AD. Physicians can make a probable diagnosis on living patients with a complete medical history, neurological and psychological tests, a physical exam, standard laboratory tests and a brainimaging scan. AD diagnosis can be made with up to $90 \%$ accuracy by experienced physicians, once symptoms begin [3,4]. In order to accomplish a diagnosis with $100 \%$ certainty, examination of brain tissue after death is needed [5].

It is important to obtain an early and accurate diagnosis of $\mathrm{AD}$, since it enables to 
develop strategies for coping with the disease. Moreover, drug intervention works better in the earliest stages of the disease. In this sense, electrical activity in the brain has been widely analyzed to improve quality of diagnosis and to achieve early hallmarks of AD. Both electroencephalogram (EEG) and magnetoencephalogram (MEG) are generated by synchronous oscillations of pyramidal neurons. Nevertheless, they reflect slightly different characteristics, since EEG is sensitive to all primary currents and MEG is only affected by current flows oriented parallel to the scalp [6,7]. Other difference between EEG and MEG arises from the insensitivity of magnetic fields to inhomogeneities of the head. Electrical activity is more affected than magnetic oscillations by skull and extracerebral brain tissues. Moreover, EEG rhythms can be significantly influenced by some technical and methodological issues, like distance between electrodes, sensor placement or reference point. Thus, MEG can be seen as a complementary signal to EEG, which is less distorted by head structures, provides reference-free recordings and offers higher spatial resolution than conventional electroencephalography [7].

In relation to the aforementioned electromagnetic recordings, both EEG and MEG background oscillations exhibit several abnormalities in AD (for recent reviews, we refer to [8] and [9]). Hence, in moderate and severe AD patients an increase of the power in the low frequency bands (i.e., delta and theta) and a decrease in the high frequency bands (i.e., alpha and beta) have been observed, both in EEG [10-13] and MEG studies [14,15]. Coherence values of the alpha and beta bands also decrease in $\mathrm{AD}$ patients when compared with controls using EEG [16,17]. This result is supported by some MEG studies, where a decreased coherence in the alpha band has been found [18]. However, a general decrease of coherence values in all frequency bands has been reported when analyzing MEG recordings [14]. On the other hand, nonlinear studies suggest that electromagnetic brain activity in AD is globally less complex and more regular than in elderly controls [19-25]. Nevertheless, some authors 
indicate that the reduction in MEG complexity depends on the frequency band [26]. It should be pointed out that nonlinear properties of electromagnetic recordings are associated with their linear characteristics (e.g. the relative power spectrum) [8]. Hence, an increase in low frequency oscillations might produce a wrongly decreased dynamical complexity [9]. To avoid this incorrect interpretation, surrogate data testing can be employed. Briefly, the method constructs a surrogate time series with the same linear properties as the original one, but varying its nonlinear structure. Then, the nonlinear parameter is calculated for each time series and, finally, they are compared. In this way, Jelles et al. [27] used the surrogate data method with EEG recordings to compute the correlation dimension. They found no change in the nonlinear structure of the EEG in very mild AD patients and elderly controls.

Several studies have also analyzed the so called slowing of the spontaneous EEG/MEG activity using spectral based measures. They characterize the shape of the power spectrum. A power spectrum shifted to the left has been observed in AD patients' EEG [28,29], along with an absence of a dominant activity from 6.5 to $12 \mathrm{~Hz}[30,31]$. The topography of the EEG power spectrum in $\mathrm{AD}$ has also been studied by means of power measures [32] and using several spectral parameters like mean frequency [10,11,13,28,33,34], peak frequency $[11,28,33,35]$ and transition frequency [35]. While the EEG has been broadly analyzed using spectral parameters, only a few studies have investigated the MEG spectral patterns in AD. Studies analyzing spontaneous MEG recordings have employed mean frequency [36] and peak frequency [14, 15]. Their results are in accordance with previous EEG studies reporting a decrease in characteristic frequencies in $\mathrm{AD}$, which was accentuated by the severity of the disease.

The aforementioned studies analyzed raw signals (i.e. the recorded MEG brain activity). However, some attempts have been made to reconstruct the sources which generate these recordings. Thus, works counting sources have also found differences between AD 
patients and controls [15,37]. Osipova et al. [15] proposed that MEG slowing might be due to an increase in activation of low frequency oscillators rather than slowing of existing sources. In this sense, Fernández et al. [37] observed an enhanced value in dipole density in the delta and theta bands of MEG background activity in $\mathrm{AD}$. This result could be related to the increase of slow rhythms in AD. Source reconstruction studies are closely related to those analyzing MEG recordings. Nevertheless, it should be noticed that they studied different characteristics of the oscillatory brain activity. Reconstructed sources reflect the internal generators of MEG activity, whereas raw signals represent the oscillatory activity generated by these sources. Hence, they can be viewed as complementary analyses which can help to understand the brain dynamics in $\mathrm{AD}$.

From another point of view, power spectral density (PSD) has also been used to extract markers of correlation with anaesthetic drug effect. Thus, an estimation of the spectral content of PSD, called 95\% spectral edge frequency, has been studied to monitor depth of anaesthesia $[38,39]$. Similarly, parameters from the context of nonlinear dynamics have also been employed to yield additional information to linear spectral based measures. One of them is the spectral entropy, which represents the Shannon entropy computed over the normalized power spectral density function [38-40].

In this study, we analyzed spontaneous MEG recordings from AD patients and healthy controls to identify discriminating features between both groups. Hence, we calculated five spectral parameters (mean frequency, individual alpha frequency, transition frequency, 95\% spectral edge frequency and spectral entropy), which could help to summarize the power spectrum. While a few MEG studies have analyzed individually some of these indexes as AD markers (i.e., mean frequency and individual alpha frequency), we calculated simultaneously the aforementioned five spectral parameters. Therefore, we achieved a complete description of the PSD function. 


\section{Materials}

\subsection{Selection of subjects}

Twenty patients $(7$ men and 13 women, age $=73.05 \pm 8.65$ years, mean \pm standard deviation SD), recruited from the Asociación de Familiares de Enfermos de Alzheimer (AFAL), participated in the study. To obtain the clinical diagnosis, exhaustive medical, physical, neurological, psychiatric and neuropsychological examinations were performed. Tests were complemented with brain scans to exclude other causes of dementia and with Mini-Mental State Examination (MMSE) to evaluate the degree of dementia [41]. The mean MMSE score for the patients was $17.85 \pm 3.91$ (mean \pm SD). All patients fulfilled the criteria of probable $\mathrm{AD}$ according to the clinical guidelines of the National Institute of Communicative Disorders and Stroke and the $\mathrm{AD}$ and Related Disorders Association (NINCDS-ADRDA) [42]. In addition, AD patients did not suffer from other medical, neurological and psychiatric diseases, and were not taking any medication that could significantly affect MEG recordings (cholinesterase inhibitors, benzodiazepines or antidepressives).

Twenty-one healthy elderly subjects $(9$ men and 12 women, age $=70.29 \pm 7.07$ years, mean \pm SD) formed the control group. They were volunteers with no history of neurological or psychiatric disorders. The mean MMSE score was $29.10 \pm 1.00$ points. It should be noted that their mean age was not significantly different from the mean age of AD patients $(p>$ $0.05)$.

All control subjects and all caregivers of the demented patients gave their consent to participate in the study, which was approved by the local ethics committee. 


\subsection{MEG data collection}

Data recording was performed using a 148-channel whole-head magnetometer (MAGNES $2500 \mathrm{WH}, 4 \mathrm{D}$ Neuroimaging). The register device was located in a magnetically shielded room in the Centro de Magnetoencefalografía Dr. Pérez-Modrego, Spain. In order to avoid segments contaminated with artefacts, MEG recordings were obtained with subjects in a relaxed state, awake and with eyes closed. In order to avoid and control drowsiness effects, subjects' behaviour and level of consciousness were controlled during the whole recording by means of a video-camera. Hence, five minutes of MEG background activity were recorded at a sampling rate of $678.17 \mathrm{~Hz}$. Data were filtered using both a $0.1-200 \mathrm{~Hz}$ hardware bandpass filter and a $50 \mathrm{~Hz}$ notch filter. To reduce data length, the recordings were downsampled by a factor of four, obtaining a sampling rate of $169.54 \mathrm{~Hz}$.

The recordings were contaminated by both low and high-frequency artefacts. Whereas, low-frequency artefacts were typically due to eye movements, high-frequency artefacts were usually generated by muscle activity. An experienced physician, who was blind to the patients' diagnosis, selected artefact-free time segments by visual inspection. Signals were arranged in consecutive 10-s artefact free epochs, obtaining a mean of $26.4 \pm 5.5$ fragments (mean \pm SD) per subject and per channel. Finally, each segment of 1696 samples was processed with a $0.4-70 \mathrm{~Hz}$ digital bandpass filter.

\section{Methods}

\subsection{Spectral analysis and definition of parameters}

To characterize the spectral content of each MEG recording, we used the Fourier transform and calculated five parameters. Initially, we computed the power spectral density (PSD) for each MEG epoch, which was estimated from the Fourier transform of the autocorrelation function. Then, we obtained the mean PSD per channel and subject from $1 \mathrm{~Hz}$ 
to $64 \mathrm{~Hz}$.

The first estimated index was the mean frequency (MF), which offers a simple means of summarizing the whole spectral content of PSD. It is defined as the frequency which comprises $50 \%$ of the power. This issue is represented in the equation 1 , where MF is numerically calculated from the discrete PSD between $1 \mathrm{~Hz}$ to $64 \mathrm{~Hz}$. It should be noticed that the discrete PSD was computed as the Fourier transform of the biased estimate of the autocorrelation function. Thus, if the raw signal contains $N$ samples, then the autocorrelation function is a vector of length $2 \mathrm{~N}-1$. Due to this fact, the spectral resolution was $0.05 \mathrm{~Hz}$ in the present study.

$$
0.5 \cdot \sum_{1 H z}^{64 H z} P S D(f)=\sum_{1 H z}^{M F} P S D(f)
$$

Then, we computed the individual alpha frequency (IAF), also named peak frequency. Alpha oscillations are dominant in the EEG of resting normal subjects, with the exception of irregular activity in the delta band and lower frequencies [43]. This issue involves that the PSD displays a peak around the alpha band $(8-13 \mathrm{~Hz})$. To detect the IAF, we calculated the mean frequency in the extended alpha band (4-15 Hz), since we observed several peaks in this range [35]. This is shown in the next equation.

$$
0.5 \cdot \sum_{4 H z}^{15 H z} P S D(f)=\sum_{4 H z}^{I A F} P S D(f)
$$

Previous EEG studies estimated the so called transition frequency (TF), as the minimum power in the theta band [35]. This index is strongly associated to the IAF. In this work, we computed the TF as the mean frequency from $1 \mathrm{~Hz}$ to the IAF as it is shown in 
equation 3. Hence, we obtained an estimation of the minimum in the delta and theta range that is not affected by local minima and takes into account the contribution of the delta band.

$$
0.5 \cdot \sum_{1 H z}^{I A F} P S D(f)=\sum_{1 H z}^{T F} P S D(f)
$$

Similar to the MF, the 95\% spectral edge frequency (SEF95) is estimated as the frequency which comprises $95 \%$ of the power. Although it was also possible to calculate the 90\% spectral edge frequency (SEF90), some authors have indicated that SEF95 is more stable and reliable than SEF90 [44]. Thus, we used SEF95 as an indicator of the upper limit in the spectral content of PSD. Equation 4 represents the expression from which the SEF95 is calculated analogously to the previous parameters.

$$
0.95 \cdot \sum_{1 H z}^{64 H z} P S D(f)=\sum_{1 H z}^{S E F 95} P S D(f)
$$

Finally, the spectral entropy (SE) was estimated to measure the flatness of PSD. It represents a disorder measure, which can be used as an estimation of MEG irregularity [45]. Thus, a uniform spectrum with a broad spectral content (e.g. white noise) yields a high SE value, whereas a more predictable signal that contains only a few spectral components (e.g. a sum of sinusoids) gives a low SE value [46]. To calculate SE, we applied the definition of Shannon's entropy computed over the normalized PSD function [45]. This is stated in equation 5, where $\operatorname{PSD}_{n}(f)$ denotes the normalized PSD from $1 \mathrm{~Hz}$ to $64 \mathrm{~Hz}$.

$$
S E=-\sum_{f=1 H z}^{64 H z} P S D_{n}(f) \cdot \log \left[P S D_{n}(f)\right]
$$


All features were log-transformed to minimize problems with skewness and kurtosis [47]. Thus, we obtained parameters with a distribution closer to a normal curve.

\subsection{Principal Component Analysis (PCA)}

After the estimation of the parameters, we had 148 values per subject, since MEG recordings proceeded from 148 channels. Due to the high spatial density of sensors, it is possible to hypothesize that there exists some redundancy. Therefore, the use of principal component analysis (PCA) could help to summarize information and detect correlations among our variables.

In order to detect redundancy, PCA identifies orthogonal directions of maximum variance in the original data. Then, they are projected into a lower-dimensionality space formed by a subset of the highest variance components. The new uncorrelated variables are called principal components and are ordered by decreasing variance. Hence, the first principal component accounts for as much of the variability in the data as possible, and each successive component explains as much of the remaining variability as possible. To determine the principal components that should be retained for further analysis, we can choose among several methods. We can use the broken stick model, Velicer's partial correlation procedure, cross-validation, Bartlett's test for equality of eigenvalues, Kaiser's criterion, Cattell's scree test and cumulative percentage of explained variance, among others $[48,49]$. Nevertheless, the selection of a complex criterion does not involve a significant improvement in most cases, as some authors have indicated [50]. Thereby, a simple selection rule can work just as well as an elaborated scheme. 


\subsection{Statistical analysis}

We studied the differences between groups by means of Student's t-test. Normality of distribution was assessed with Lilliefors test, whereas homoscedasticity was analyzed with Levene's test.

We used notched boxplots to visualize the distribution of each parameter. This is a graph for descriptive statistics, which divides the ordered values of the data into four 'equal' parts, separated by three horizontal lines. The sample median is displayed as a line approximately in the middle of the box. Deviation of the distribution from symmetry is indicated by a shift of the median from the middle of the box. The 25 th and 75 th percentiles of the sample are established as the lower and upper lines of the box, respectively. The notches included in the box represent a graphic confidence interval about the median of a sample. In addition, boxplots show two lines extending above and below the box, which are called 'whiskers'. They are drawn from each end of the box to the farthest observation outside the box extended to a maximum of 1.5 times the interquartile range. Any values beyond these whiskers are defined as data outliers and marked by the symbol, '+' [51].

Receiver Operating Characteristics (ROC) plots help us to assess the ability of the parameters to distinguish between groups [52]. ROC plots are graphs of sensitivity against 1specificity. The sensitivity represents the percentage of patients correctly classified and specificity is the proportion of controls properly identified. We also calculated the point closest to the left top corner of the graph (i.e. $100 \%$ sensitivity and $100 \%$ specificity) and marked it with the symbol ' $\$$ '. It indicates the optimum threshold that maximizes the accuracy, which is defined as the total number of patients and controls correctly recognized. Finally, we also employed the area under ROC curve (AUC) as a complementary measure of parameters' performance to discriminate between both groups. 


\section{Results}

Initially, we calculated the PSD for each $10 \mathrm{~s}$ epoch in the 148 channels. A mean PSD for each channel and each subject was obtained averaging the PSD functions of the different epochs. Figure 1 depicts the average relative power spectra for both groups. As it can be seen, the spectrum of $\mathrm{AD}$ patients is shifted to the left in comparison with controls. This fact supports the notion of EEG/MEG slowing in AD. The spectral parameters defined in the previous section were then computed from the aforementioned PSD functions. Table 1 displays their mean values averaged for each group and for all channels, whereas figure 2 depicts the notched boxplots with their distributions. AD patients showed frequencies (i.e., MF, IAF, TF and SEF95) significantly lower than control subjects, which points out the slowing of spontaneous MEG brain activity. In the case of spectral entropy, AD patients also obtained significantly lower mean values than healthy controls. This fact indicates that MEG background rhythms are less irregular in AD patients than in control subjects' MEGs. In this

sense, figure 1 shows a broader spectral content for control subjects than for AD patients. According to the definition of SE, AD patients' MEG is a more predictable signal than magnetic brain oscillations of control subjects.

Insert Table 1 and Figures 1 and 2 around here

Afterwards, parameters were log-transformed and PCA was applied. The percentage of variance, together with the accumulated percentage of variance explained by the first 5 principal components are shown in table 2. It should be noted that the first principal component retained between $75.46 \%$ of variance in the TF parameter and $81.48 \%$ in the MF parameter. On the other hand, it should also be pointed out that the accumulated percentage of variance explained by the 5 first principal components reaches $91 \%$ for all parameters. These 
values indicate that there exists a great amount of redundancy in the data of the 148 channels. In this way, PCA can help to reduce the dimensionality of the problem with a minimum loss of information. We retained only the first principal component for every parameter, since it explained more than $75 \%$ of the variance for all of them. Thus, the analysis scheme is simplified, at the same time that the interpretation of the results is easier than taking the 148 values per subject simultaneously.

Insert Table 2 around here

We analyzed the differences between the transformed features and calculated four statistics from the ROC plots (i.e., sensitivity, specificity, accuracy and AUC). Results are shown in table 3, while ROC plots are displayed in figure 3. It should be noticed that points marked in ROC plots represent sensitivity against 1-specificity for each threshold. The symbol ' $\checkmark$ identifies the points with the maximum accuracy, which appear in table 3 . The lowest $p$-value and the highest AUC were obtained with MF, a result that suggests MF showed the best global performance $(\mathrm{AUC}=0.912$, sensitivity $=85.00 \%$, specificity $=$ $85.71 \%$ and accuracy $=85.37 \%$ ). The $p$-value and AUC for SE were slightly lower than in MF $($ AUC $=0.888$, sensitivity $=90.00 \%$, specificity $=76.19 \%$ and accuracy $=82.93 \%)$. It should be noted that both MF and SE use the information of the broad spectrum in its definition. With regard to IAF and TF, the statistical differences were not as significant as those reached using MF. In this sense, we achieved a lower $p$-value, together with a higher AUC $($ AUC $=0.821$, sensitivity $=80.00 \%$, specificity $=80.95 \%$ and accuracy $=80.49 \%$ ), with IAF than with TF (AUC $=0.760$, sensitivity $=70.00 \%$, specificity $=76.19 \%$ and accuracy $=73.17 \%$ ). The lowest classification parameters $($ AUC $=0.698$, sensitivity $=$ $65.00 \%$, specificity $=66.67 \%$ and accuracy $=65.85 \%$ ) were obtained with SEF95. 
Insert Table 3 and Figure 3 around here

Finally, a stepwise discriminant analysis was performed to assess the combination of parameters which reached the best classification performance. Table 4 shows the variable groups that obtain an overall accuracy greater than the individual parameters. It should be noted that combinations of two parameters do not improve the accuracy of MF, but three or four spectral measures are needed. In a similar sense, the group of the five spectral indexes only reaches $85.37 \%$ of accuracy. With regard to the parameters selected in the stepwise discriminant analysis, both TF and SEF95 appear in almost all the combinations. Although these measures do not provide the best individual classification statistics, results point out that they contribute to improve the classification rates.

Insert Table 4 around here

\section{Discussion}

In this study, we compared the ability of several spectral based measures to distinguish between MEG background activity from 20 AD patients and 21 healthy controls. By using the PSD function, we estimated five spectral parameters (MF, IAF, TF, SEF95 and SE). Prior to statistical analysis, we reduced the dimensionality of the problem by using PCA, since MEG signals were recorded from 148 channels simultaneously. We selected the first principal component for each spectral parameter, as it explained more than $75 \%$ of the variance. Finally, the performance of each spectral index was assessed using several Student's t-tests and ROC curves. The best results - the most significant differences and the highest global 
classification statistics - were obtained with MF. SE also showed significant differences with an AUC close to that obtained with MF. On the other hand, the differences between both groups using IAF and TF were less significant as compared to MF. Nevertheless, IAF showed a lower $p$-value and higher AUC than TF. Finally, the highest $p$-value, along with the lowest AUC, was obtained with SEF95. When we combined the parameters to augment the classification rate, we found that only variable groups of three or four indexes provided accuracies greater than MF. Moreover, both TF and SEF95 played an important role to improve classification statistics, as they appeared in almost all the optimal combinations.

Further inspection of results points out that MF in AD patients' MEGs is significantly lower than in healthy controls' MEGs. Hence, averaged $\mathrm{MF}$ values for each group are, $\mathrm{MF}_{\mathrm{AD}}$ $=6.63 \pm 3.01 \mathrm{~Hz}$ and $\mathrm{MF}_{\mathrm{C}}=12.17 \pm 3.87 \mathrm{~Hz}$ (henceforth all values are expressed as mean \pm SD). These findings are in agreement with previous studies, which also analyzed spontaneous MEG signals in AD. Fernández et al. [36] reported a significant decrease in the mean frequency of $\mathrm{AD}$ patients and controls. As previously mentioned, MF of EEG background activity also decreases significantly in $\mathrm{AD}$ when compared with healthy controls $[10,11,13,28,33,34]$. With regard to the obtained mean values, they were strongly influenced by the frequency band used in the MF estimation, which was usually lower than in our study. Moreover, although EEG and MEG are related, they do not reflect identical information [7].

Significant MEG slowing can also be observed when analyzing the IAF. We obtained averaged IAF values, $\mathrm{IAF}_{\mathrm{AD}}=7.97 \pm 1.15 \mathrm{~Hz}$ and $\mathrm{IAF}_{\mathrm{C}}=9.40 \pm 0.69 \mathrm{~Hz}$. The mean values are in accordance with those obtained in other studies using MEG signals at rest. In this way, Osipova et al. [15] analyzed the brain activity in 11 AD patients and 12 elderly controls, reporting the mean values, $\mathrm{IAF}_{\mathrm{AD}}=8.50 \pm 1.36 \mathrm{~Hz}$ and $\mathrm{IAF}_{\mathrm{C}}=9.50 \pm 1.25 \mathrm{~Hz}$. Their results did not show significant differences $(p>0.05)$, and the IAF in AD patients was slightly higher than in our study. A possible explanation for this fact may be that they analyzed MEG signals 
from patients with a mean MMSE score of $20.8 \pm 4.0$. This value is slightly higher than our mean MMSE, indicating a less severe degree of dementia. In addition, they estimated the alpha peak as the maximum frequency in the alpha range, whereas we computed the IAF as the mean frequency from $4 \mathrm{~Hz}$ to $15 \mathrm{~Hz}$ to avoid the detection of local maxima [43]. Berendse et al. [14] also calculated the IAF of spontaneous MEG activity as the maximum in the extended alpha range. Nevertheless, the mean values they obtained were, $\mathrm{IAF}_{\mathrm{AD}}=7.50 \mathrm{~Hz}$ and $\mathrm{IAF}_{\mathrm{C}}=$ $10.80 \mathrm{~Hz}$. Their results are different to our mean values and those obtained by Osipova et al. [15]. This can be due to the fact that Berendse et al. [14] only analyzed MEG signals from 5 AD patients and 5 controls, which represents a considerably small population. Regarding to the severity of AD subjects, they enrolled patients with a mean MMSE score of 19.8. The IAF has also been calculated in previous EEG studies analyzing brain activity at rest $[11,28,31,33,35]$. The obtained results showed a reduction in the frequency of the alpha peak, when it was calculated as the maximum in the alpha band $[11,31,35]$, as well as the mean frequency in the alpha range $[28,33]$.

TF is closely related to IAF, since it is used in its definition. Our findings also indicate a significant slowing of the $\mathrm{TF}$ in $\mathrm{AD}, \mathrm{TF}_{\mathrm{AD}}=3.70 \pm 1.40 \mathrm{~Hz}$ and $\mathrm{TF}_{\mathrm{C}}=4.88 \pm 1.23 \mathrm{~Hz}$. This fact is in disagreement with the results obtained in a study analyzing EEG background activity of AD patients and elderly subjects, where no significant reduction in TF was reported [35]. Moretti et al. [35] obtained mean values of $\mathrm{TF}$ for each group, $\mathrm{TF}_{\mathrm{AD}}=5.30 \pm 1.07 \mathrm{~Hz}$ and $\mathrm{TF}_{\mathrm{C}}$ $=5.40 \pm 0.80 \mathrm{~Hz}$. It can be observed that TF for controls is similar to our findings, but for AD patients the values clearly differ. This might be due to the fact that AD patients were less severely demented, according to their mean MMSE score $(20.58 \pm 0.28)$. Moreover, the way to calculate the TF differs from the estimation of TF that we employed. As previously mentioned, we computed TF as the mean frequency from $1 \mathrm{~Hz}$ to IAF in order to avoid local minima and to take into account the contribution of the delta band. It should also be noticed that in this 
case comparisons between both studies should be made with care, since we used MEG recordings while Moretti et al. [35] employed EEG signals.

Although SEF95 has been successfully employed to monitor depth of anaesthesia [3840], our results are less significant than those obtained with MF, IAF, TF and SE. This finding seems to indicate that there is little change in the spectral content at high frequencies when comparing AD patients and control subjects. Nevertheless, it should be noted that AD patients showed lower mean values than controls with SEF95, SEF95 $5_{\mathrm{AD}}=31.05 \pm 8.73 \mathrm{~Hz}$ and $\mathrm{SEF95}$ $=37.03 \pm 6.95 \mathrm{~Hz}$. This fact supports the notion of slowing in the MEG spectrum of AD patients in comparison to elderly subjects, which was observed with the previous parameters.

The SE yielded significant differences, similar to those obtained using the MF. The AD patients' mean SE values $(0.83 \pm 0.07)$ were lower than in controls $(0.89 \pm 0.03)$. According to the definition of SE, these results suggest that AD patients have a less irregular spectral distribution than healthy subjects [45]. This result should be interpreted in terms of the flatness of the frequency spectrum, i.e. AD patients have a less uniform spectral content than controls, as it is shown in figure 1. Previous studies analyzing MEG recordings at rest found a global loss of complexity in $\mathrm{AD}$ by using the Lempel-Ziv complexity, which represents the chance of new pattern generation in a discrete sequence [25]. Nevertheless, the loss of complexity was only observed in the high frequencies (14 Hz to $40 \mathrm{~Hz}$ ), when individual frequency bands were analyzed [26]. In this case, van Cappellen van Walsum et al. [26] used the neural complexity to capture regularities based on the deviation from independence among subsets of a system. Abásolo et al. [22,24] reported a reduction of irregularity in AD patients' EEG using SE, approximate entropy [22] and sample entropy [24]. Their results with SE were not significant $(p>0.05)$. On the other hand, significant differences were observed with both sample entropy and approximate entropy $(p<0.01)$. It should be noted that both approximate entropy (ApEn) and sample entropy (SampEn) are 
embedding entropies. While ApEn computes the logarithmic likelihood that runs of patterns that are close (within $r$ ) for $m$ contiguous observations remain close (within the same tolerance width $r$ ) on subsequent incremental comparisons, SampEn measures the selfsimilarity in the time series and reduces the bias that introduces ApEn [24]. Decreased complexity in EEG background activity has also been observed analyzing several nonlinear measures as the Lempel-Ziv complexity [23], the first Lyapunov exponent [19] and the correlation dimension $[19,20]$. The first Lyapunov exponent is a measure of flexibility of information processing of the brain, whereas the correlation dimension reflects the number of independent variables that are necessary to describe the dynamics of the system [8]. Finally, it should be noted that these nonlinear measures may be wrongly influenced by the slowing of the power spectrum [9]. To avoid an incorrect interpretation of the parameters, a few EEG studies have employed surrogate data testing, exploring the nonlinearity of the EEG in AD. Thus, Jelles et al. [27] observed a more complex behaviour of AD patients when compared with controls, in terms of the correlation dimension. However, further works are needed to obtain more general conclusions.

Frequency parameters have shown a slowing of spontaneous MEG activity in AD. In this sense, some authors point out that the cholinergic system modulates the spontaneous cortical activity at the theta and alpha bands, along with the functional coupling in the theta band [53]. Given that cholinergic deficit involves a loss of the neurotransmitter aceltylcholine, this fact can be partly responsible of MEG slowing in AD. Nevertheless, AD is a heterogeneous disease, and therefore, it may not be sufficient to explain the different alterations of the magnetic brain activity.

It should be noted that the origin of the observed MEG slowing could be drug-related. We discarded this issue because to none of the patients were taking any medication which could affect electromagnetic brain activity at the moment of the recording. In a similar sense, 
both EEG and MEG slowing have also been reported in other neurodegenerative diseases like mild cognitive impairment [13,36], vascular dementia [28,35,54], Lewy body dementia [18,31], major depression [34,55,56], dementia associated with Parkinson's disease [31,57,58], Pick's disease [59], Huntington's chorea [60] and progressive supranuclear palsy [31]. To extract accurate markers of $\mathrm{AD}$, further studies should analyze differences in the slowing of EEG/MEG rhythms when comparing $\mathrm{AD}$ and the aforementioned diseases.

Other limitation to comment is related with the small sample size that we employed, which determines the power of the statistical test. A small sample size increases beta (i.e., the probability of generating a false negative, or a type II error), while at the same time the power of the test decreases (i.e., 1-beta). Given that we fixed alpha to 0.05 (i.e., the probability of making a false positive, or a type I error), the only way to minimize beta is to increase the sample size.

Finally, it should be mentioned that by using PCA analysis we selected only one principal component for each parameter. This involves a loss of spatial information, which could be partially avoided by computing average spectra for a number of brain regions. Then, a repeated measures ANOVA with 'Group' and 'Region' as factors, could be performed to analyze the statistical significance. Nevertheless, when we use this approach we should take into account that a recording channel does not necessarily measure only the brain oscillations under that sensor, but it can reflect activity from other regions. Hence, to avoid an incorrect interpretation of results in terms of spatial patterns, we should perform some kind of source analysis.

\section{Conclusions}

As previously mentioned, only a few MEG studies have explored the ability of spectral indexes as $\mathrm{AD}$ descriptors. Both $\mathrm{MF}$ and IAF have been analyzed individually in 
previous works $[14,15,36]$. Nevertheless, to obtain a reliable description of the power spectrum, it might be more convenient to employ a set of measures, instead of summarizing the whole content of PSD using a single index. In this sense, to the best of our knowledge, this study may be considered the first attempt to characterize the power spectrum of spontaneous MEG activity in AD patients and healthy controls using all the aforementioned spectral parameters simultaneously. Our results support the notions that AD involves both a slowing and an irregularity reduction of electromagnetic brain activity when compared with elderly subjects.

It should also be noted that future efforts will be directed to increase the sample size, as well as to extend the results to other neurodegenerative diseases. In a similar way, further works will be addressed to obtain a minimal loss of spatial information by performing source analysis and using other dimensionality reduction techniques.

Summarizing, our findings suggest that a complete spectral description of the spontaneous MEG activity may help to obtain useful markers of AD contributing, at the same time, to the understanding of the disease.

\section{Acknowledgements}

This work has been partially supported by the Ministerio de Educación y Ciencia and FEDER grant MTM 2005-08519-C02-01, and the grant project from Consejería de Educación de la Junta de Castilla y León VA108A06. The authors would like to thank the Asociación de Enfermos de Alzheimer (AFAL) for supplying the patients who have participated in our study. 


\section{References}

[1] Bird TD. Alzheimer's disease and other primary dementias. In: Braunwald E, Fauci AS, Kasper DL, Hauser SL, Longo DL, Jameson JL, editors. 'Harrison's Principles of Internal Medicine'. New York: The McGraw-Hill Companies Inc, 2001:2391-2399.

[2] Katzman R. Current research on Alzheimer's disease in a historical perspective. In: Khachaturian ZS, Radebaugh TS, editors. 'Cause(s), Diagnosis, Treatment and Care'. Boca Raton, FL: CRC Press, 1996:15-29.

[3] Larson EB, Edwards JK, O'Meara E, Nochlin D, Sumi SM. Neuropathologic diagnostic outcomes from a cohort of outpatients with suspected dementia. J Gerontol 1996;51:M313-M318.

[4] Rasmusson DX, Brandt J, Steele C, Hedreen JC, Troncoso JC, Folstein MF. Accuracy of clinical diagnosis of Alzheimer disease and clinical features of patients with nonAlzheimer neuropathology. Alzheimer Dis Assoc Disord 1996;10:180-188.

[5] Rossor M. Alzheimer's disease. In: Donaghy M, editor. 'Brain's Diseases of the Nervous System'. Oxford: Oxford University Press, 2001:750-754.

[6] Hämäläinen M, Hari R, Ilmoniemi RJ, Knuutila J, Lounasmaa OV. Magnetoencephalography - theory, instrumentation, and applications to non-invasive studies of the working human brain. Rev Mod Phys 1993;65:413-497.

[7] Hari R. Magnetoencephalography in clinical neurophysiological assessment of human cortical functions. In: Niedermeyer E, Lopes da Silva F, editors. 'Electroencephalography: Basic Principles, Clinical Applications, and Related Fields'. Phyladelphia: Lippincott Williams \& Wilkins, 2005:1165-1197.

[8] Jeong J. EEG dynamics in patients with Alzheimer's disease. Clin Neurophysiol 2004;115:1490-1505. 
[9] Stam CJ. Nonlinear dynamical analysis of EEG and MEG: review of an emerging field. Clin Neurophysiol 2005;116:2266-2301.

[10] Coben LA, Danzinger WL, Storandt M. A longitudinal EEG study of mild senile dementia of Alzheimer type: changes at 1 year and at 2.5 years. Electroenceph clin Neurophysiol 1985;61:101-112.

[11] Soininen H, Partanen J, Laulumaa V, Helkala E-L, Laakso M, Riekkinen PJ. Longitudinal EEG spectral analysis in early stage of Alzheimer's disease. Electroenceph clin Neurophysiol 1989;72:290-297.

[12] Schreiter-Gasser U, Gasser T, Ziegler P. Quantitative EEG analysis in early onset Alzheimer's disease: a controlled study. Electroenceph clin Neurophysiol 1993;86:1522.

[13] Jelic V, Johansson S-E, Almkvist O, Shigeta M, Julin P, Nordberg A, Winblad B, Wahlund L-O. Quantitative electroencephalography in mild cognitive impairment: longitudinal changes and possible prediction of Alzheimer's disease. Neurobiol Aging 2000;21:533-540.

[14] Berendse HW, Verbunt JPA, Scheltens Ph, van Dijk BW, Jonkman EJ. Magnetoencephalographic analysis of cortical activity in Alzheimer's disease: a pilot study. Clin Neurophysiol 2000;111:604-612.

[15] Osipova D, Ahveninen J, Jensen O, Ylikoski A, Pekkonen E. Altered generation of spontaneous oscillations in Alzheimer's disease. NeuroImage 2005;27:835-841.

[16] Besthorn C, Förstl H, Geiger-Kabisch C, Sattel H, Gasser T, Schreiter-Gasser U. EEG coherence in Alzheimer disease. Electroenceph clin Neurophysiol 1994;90:242-245.

[17] Adler G, Brassen S, Jajcevic A. EEG coherence in Alzheimer's dementia. J Neural Transm 2003;110:1051-1058. 
[18] Franciotti R, Iacono D, Della Penna S, Pizzella V, Torquati K, Onofrj M, Romani GL. Cortical rhythms reactivity in $\mathrm{AD}, \mathrm{LBD}$ and normal subjects. A quantitative MEG study. Neurobiol Aging 2006:27:1100-1109.

[19] Jeong J, Kim SJ, Han SH. Non-linear dynamical analysis of the EEG in Alzheimer's disease with optimal embedding dimension. Electroencephalogr clin Neurophysiol $1998 ; 106: 220-228$.

[20] Jeong J, Chae JH, Kim SY, Han SH. Nonlinear dynamic analysis of the EEG in patients with Alzheimer's disease and vascular dementia. J Clin Neurophysiol 2001;18:58-67.

[21] Stam CJ, van der Made Y, Pijnenburg YA, Scheltens P. EEG synchronization in mild cognitive impairment and Alzheimer's disease. Acta Neurol Scand 2003;108:90-96.

[22] Abásolo D, Hornero R, Espino P, Poza J, Sánchez CI, de la Rosa R. Analysis of regularity in the EEG background activity of Alzheimer's disease patients with Approximate Entropy. Clin Neurophysiol 2005;116:1826-1834.

[23] Abásolo D, Hornero R, Gómez C, García M, López M. Analysis of EEG background activity in Alzheimer's disease patients with Lempel-Ziv complexity and central tendency measure. Med Eng Phys 2006;28:315-322.

[24] Abásolo D, Hornero R, Espino P, Álvarez D, Poza J. Entropy analysis of the EEG background activity in Alzheimer's disease patients. Physio Meas 2006;27:241-253.

[25] Gómez C, Hornero R, Abásolo D, Fernández A, López M. Complexity analysis of the magnetoencephalogram background activity in Alzheimer's disease patients. Med Eng Phys 2006. In press

[26] van Cappellen van Walsum A-M, Pijnenburg YAL, Berendse HW, van Dijk BW, Knol DL, Scheltens Ph, Stam CJ. A neural complexity measure applied to MEG data in Alzheimer's disease. Clin Neurophysiol 2003;114:1034-1040. 
[27] Jelles B, van Birgelen JH, Slaets JPJ, Hekster REM, Jonkman EJ, Stam CJ. Decrease of non-linear structure in the EEG of Alzheimer patients compared to healthy controls. Clin Neurophysiol 1999;110:1159-1167.

[28] Signorino M, Pucci E, Belardinelli N, Nolfe G, Angeleri F. EEG spectral analysis in vascular and Alzheimer dementia. Electroenceph clin Neurophysiol 1995;94:313-325.

[29] Rodríguez G, Copello F, Vitali P, Perego G, Nobili F. EEG spectral profile to stage Alzheimer's disease. Clin Neurophysiol 1999;110:1831-1837.

[30] Signorino M, Pucci E, Brizioli E, Cacchio G, Nolfe G, Angeleri F. EEG power spectrum typical of vascular dementia in a subgroup of Alzheimer patients. Arch Gerontol Geriatr 1996;23:139-151.

[31] Pucci E, Cacchiò G, Angeloni R, Belardinelli N, Nolfe G, Signorino M, Angeleri F. EEG spectral analysis in Alzheimer's disease and different degenerative dementias. Arch Gerontol Geriatr 1998;26:283-297.

[32] Ihl R, Dierks T, Martin E-M, Frölich L, Maurer K. Topography of the maximum of the amplitude of EEG frequency bands in dementia of the Alzheimer type. Biol Psychiatry 1996;39:319-325.

[33] Penttilä M, Partanen JV, Soininen H, Riekkinen PJ. Quantitative analysis of occipital EEG in different stages of Alzheimer's disease. Electroenceph clin Neurophysiol 1985;60:1-6.

[34] Brenner RP, Ulrich RF, Spiker DG, Sclabassi RJ, Reynolds III CF, Marin RS, Boller F. Computerized EEG spectral analysis in elderly normal, demented and depressed subjects. Electroenceph clin Neurophysiol 1986;64:483-492.

[35] Moretti DV, Babiloni C, Binetti G, Cassetta E, Dal Forno G, Ferreri F, Ferri R, Lanuzza B, Miniussi C, Nobili F, Rodriguez G, Salinari S, Rossini PM. Individual analysis of 
EEG frequency and band power in mild Alzheimer's disease. Clin Neurophysiol 2004;115:299-308.

[36] Fernández A, Hornero R, Mayo A, Poza J, Gil-Gregorio P, Ortiz T. MEG spectral profile in Alzheimer's disease and mild cognitive impairment. Clin Neurophysiol $2006 ; 117: 306-314$

[37] Fernández A, Maestú F, Amo C, Gil P, Fehr T, Wienbruch C, Rockstroh B, Elbert T, Ortiz T. Focal temporoparietal slow activity in Alzheimer's disease revealed by magnetoencephalography. Biol Psychiatry 2002;52:764-70.

[38] Grouven U, Beger FA, Schultz B, Schultz A. Correlation of Narcotrend Index, entropy measures, and spectral parameters with calculated propofol effect-site concentrations during induction of propofol-remifentanil anaesthesia. J Clin Monit Comput 2004;18:231-240.

[39] Tonner PH, Bein B. Classic electroencephalographic parameters: median frequency, spectral edge frequency etc. Best Pract Res Clin Anaesthesiol 2006;20:147-159.

[40] Schwender D, Daunderer M, Mulzer S, Klasing S, Finsterer U, Peter K. Spectral edge frequency of the electroencephalogram to monitor depth of anaesthesia with isoflurane or propofol. Br J Anaesth 1996;77:179-184.

[41] Folstein MF, Folstein SE, McHugh PR. Mini-mental state. A practical method for grading the cognitive state of patients for the clinician. J Psychiatr Res 1975;12:189_ 198.

[42] McKhann G, Drachman D, Folstein M, Katzman R, Price D, Stadlan EM. Clinical diagnosis of Alzheimer's disease: report of NINCDS-ADRDA work group under the auspices of department of health and human services task force on Alzheimer's disease. Neurology 1984;34:939-944. 
[43] Klimesch W. EEG alpha and theta oscillations reflect cognitive and memory performance: a review analysis. Brain Res Rev 1999;29:169-195.

[44] Hudson RJ, Stanski DR, Saidman LJ, Meathe E. A model for studying depth of anesthesia and acute tolerance to thiopental. Anesthesiology 1986;65:19-27.

[45] Inouye T, Shinosaki K, Sakamoto H, Toi S, Ukai S, Iyama A, Katsuda Y, Hirano M. Quantification of EEG irregularity by use of the entropy of the power spectrum. Electroencephalogr clin Neurophysiol 1991;79:204-210.

[46] Sleigh JW, Steyn-Ross DA, Grant C, Ludbrook G. Cortical entropy changes with general anaesthesia: theory and experiment. Physiol Meas 2004;25:921-934.

[47] Pollock V, Schneider L, Lyness S. EEG amplitudes in healthy, late-middle-aged and elderly adults: normality of the distributions and correlations with age. Electroenceph clin Neurophysiol 1990;75:276-288.

[48] Jackson JE. A User's Guide to Principal Components. New York: John Wiley \& Sons, 1991:41-51.

[49] Ferré L. Selection of components in principal component analysis: A comparison of methods. Computat Statist Data Anal 1995;19:669-682.

[50] Jolliffe IT. Principal Component Analysis. New York: Springer, 2002:112-133.

[51] Hoaglin D, Mosteller F, Tukey J. Understanding robust and exploratory data analysis. New York: Wiley \& Sons, 2000.

[52] Zweig MH, Campbell G. Receiver-Operating Characteristic (ROC) plots: a fundamental evaluation tool in clinical medicine. Clin Chem 1993;39:561-577.

[53] Osipova D, Ahveninen J, Kaakkola S, Jääskeläinen IP, Huttunen J, Pekkonen E. Effects of scopolamine on MEG spectral power and coherence in elderly subjects. Clin Neurophysiol 2003;114:1902-1907. 
[54] Babiloni C, Cassetta E, Chiovenda P, Del Percio C, Ercolani M, Moretti DV, Moffa F, Pasqualetti P, Pizzella V, Romanid GL, Tecchio F, Zappasodi F, Rossini PM. Alpha rhythms in mild dements during visual delayed choice reaction time tasks: A MEG study. Brain Res Bull 2005;65:457-470.

[55] Prinz PN, Vitiell MV. Dominant occipital (alpha) rhythm frequency in early stage Alzheimer's disease and depression. Electroenceph clin Neurophysiol 1989;73:427432.

[56] Fernández A, Rodríguez-Palancas A, López-Ibor M, Zuluaga P, Turrero A, Maestú F, Amo C, López-Ibor JJ Jr, Ortiz T. Increased occipital delta dipole density in major depressive disorder determined by magnetoencephalography. J Psychiatry Neurosci 2005;30:17-23.

[57] Pin JC, Neau JP, Paquereau J, Rostykus C, Guehl D, Aubert I, Marillaud A, Gil R. Comparative study by computerized EEG of dementia of the Alzheimer type and Parkinson's disease with dementia. Neurophysiol Clin 1992;22:301-312.

[58] Kotini A, Anninos P, Adamopoulos A, Prassopoulos P. Low-frequency MEG activity and MRI evaluation in Parkinson's disease. Brain Topogr 2005;18:59-63.

[59] Stigsby B, Jóhannesson G, Ingvar DH. Regional EEG analysis and regional cerebral blood flow in Alzheimer's and Pick's diseases. Electroenceph clin Neurophysiol $1981 ; 51: 537-547$

[60] Streletz LJ, Reyes PF, Zalewska M, Katz L, Fariello RG. Computer analysis of EEG activity in dementia of the Alzheimer's type and Huntington's disease. Neurobiol Aging 1990;11:15-20. 


\section{Figures}

Figure 1. Mean relative power spectra of $A D$ patients $(A D)$ and healthy controls $(C)$.

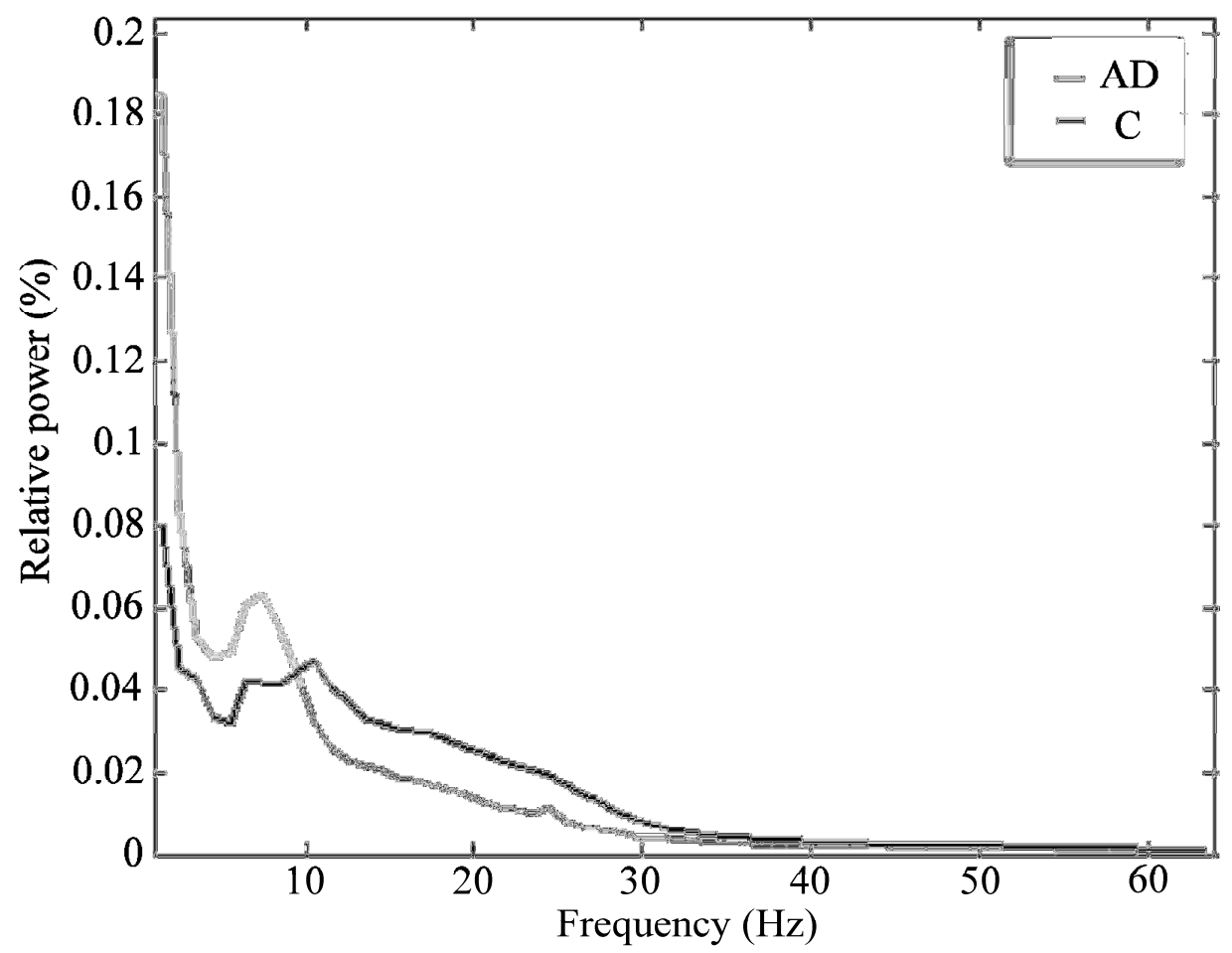


Figure 2. Notched boxplots displaying the distribution of each parameter for both groups and the corresponding $p$-values. (a) Mean frequency (MF). (b) Individual alpha frequency (IAF). (c) Transition frequency (TF). (d) 95\% spectral edge frequency (SEF95). (e) Spectral entropy (SE).

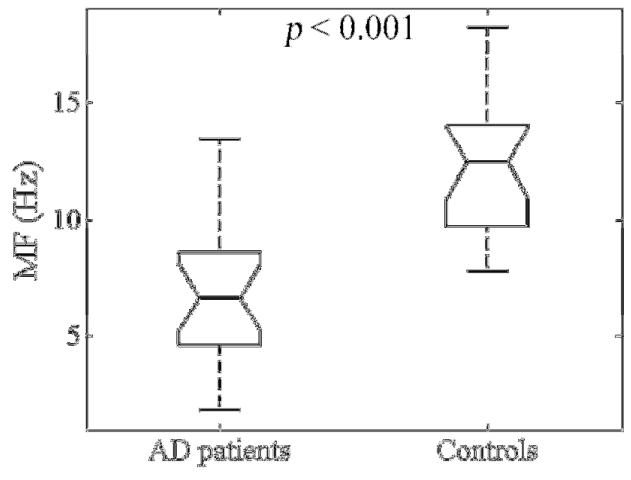

(a)

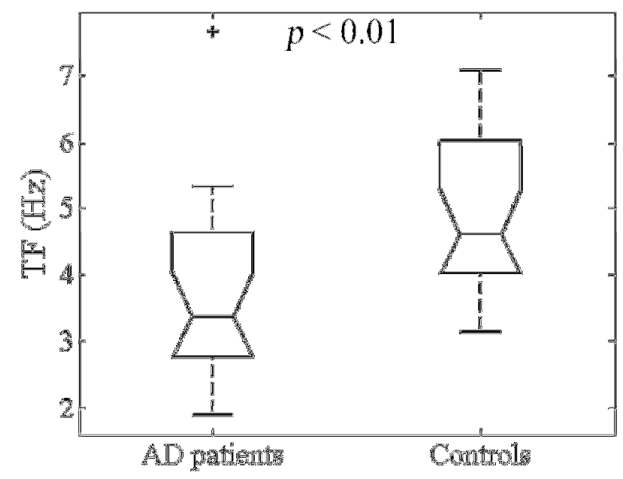

(c)

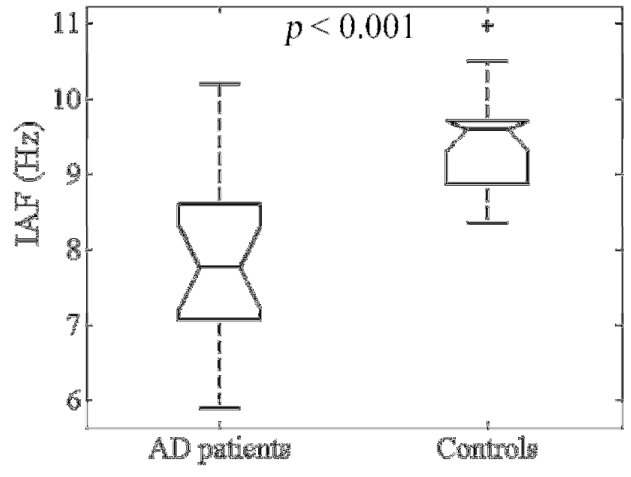

(b)

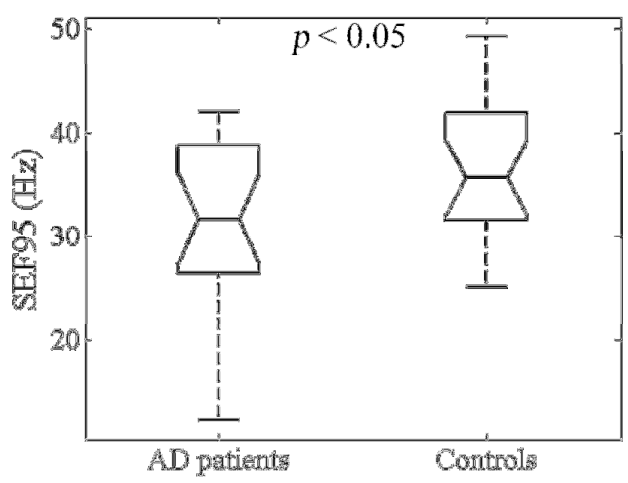

(d)

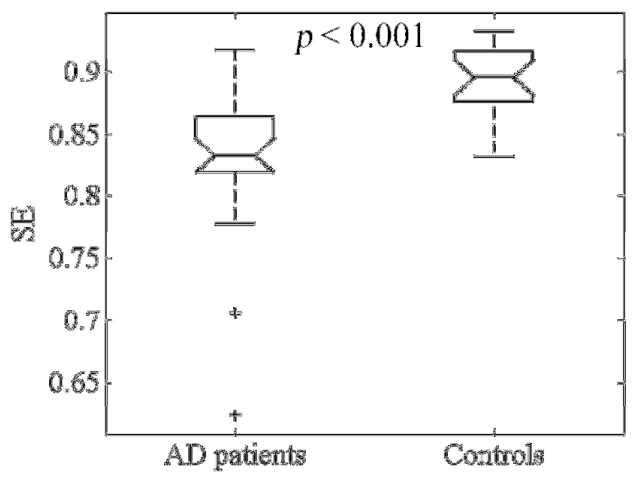

(e) 
Figure 3. ROC curves for each parameter. (a) Mean frequency. (b) Individual alpha frequency. (c) Transition frequency. (d) 95\% spectral edge frequency. (e) Spectral entropy. The ROC curve values are marked with a dot, whereas the optimum threshold is indicated with the symbol '

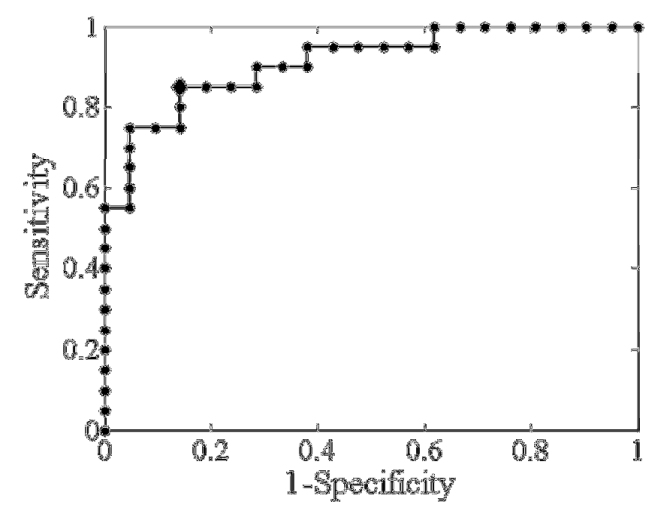

(a)

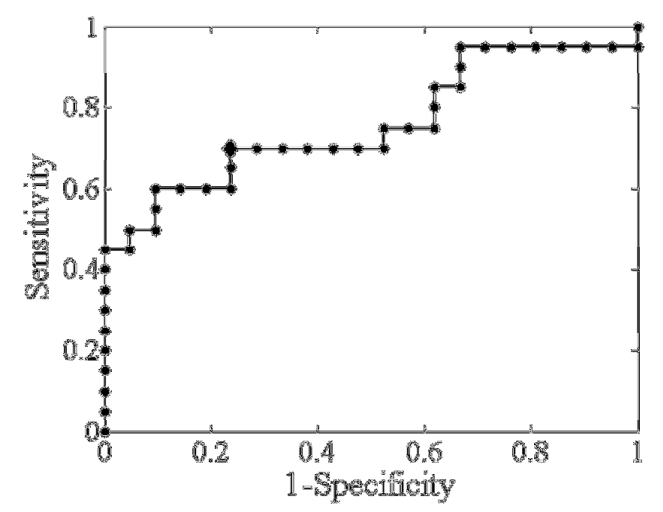

(c)

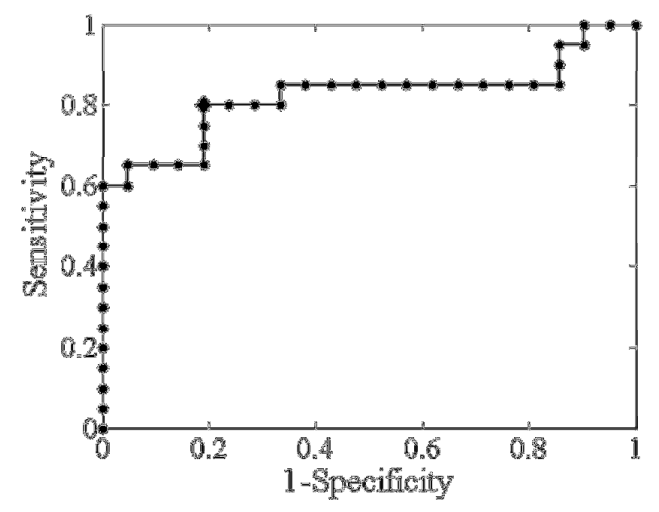

(b)

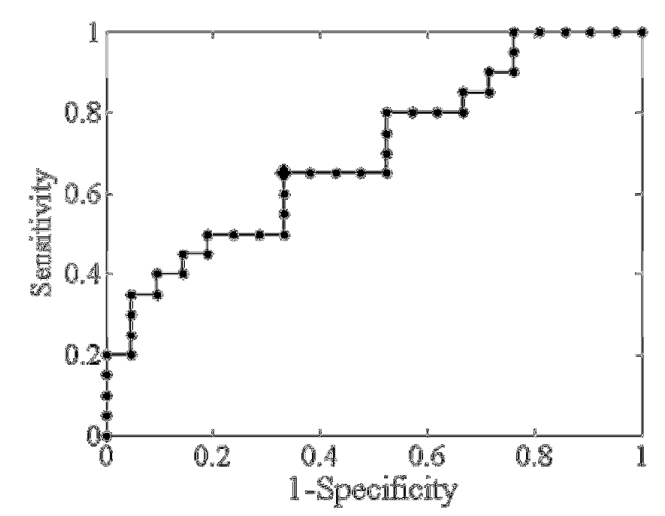

(d)

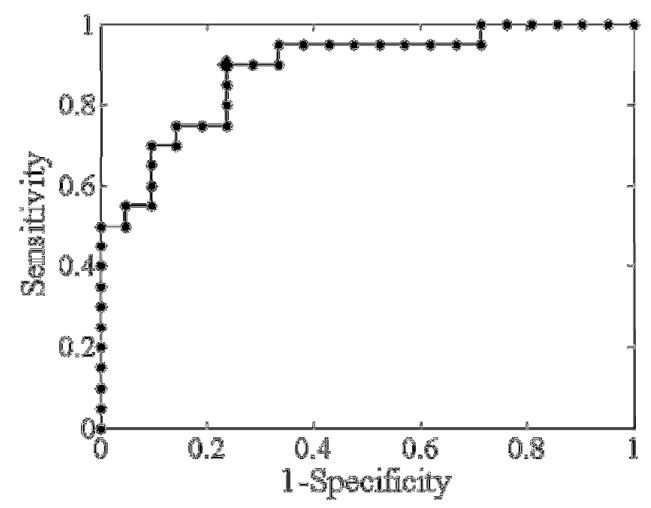

(e) 


\section{Tables}

Table 1. Averaged values for all spectral parameters.

\begin{tabular}{cccccc}
\cline { 2 - 6 } & MF $(\mathrm{Hz})$ & IAF $(\mathrm{Hz})$ & TF $(\mathrm{Hz})$ & SEF95 $(\mathrm{Hz})$ & SE \\
\cline { 2 - 6 } & Mean \pm SD & Mean \pm SD & Mean \pm SD & Mean \pm SD & Mean \pm SD \\
\hline AD patients & $6.63 \pm 3.01$ & $7.97 \pm 1.15$ & $3.70 \pm 1.40$ & $31.05 \pm 8.73$ & $0.83 \pm 0.07$ \\
Controls & $12.17 \pm 3.87$ & $9.40 \pm 0.69$ & $4.88 \pm 1.23$ & $37.03 \pm 6.95$ & $0.89 \pm 0.03$ \\
\hline
\end{tabular}

Table 2. Percentage of variance (\% Var.), together with the accumulated percentage of variance (\% Acc.) explained by the first 5 principal components for all parameters: mean frequency (MF), individual alpha frequency (IAF), transition frequency (TF), 95\% spectral edge frequency (SEF95) and spectral entropy (SE).

\begin{tabular}{|c|c|c|c|c|c|c|c|c|c|c|}
\hline \multirow{3}{*}{ Parameters } & \multicolumn{10}{|c|}{ Principal components } \\
\hline & \multicolumn{2}{|c|}{$1^{\mathrm{st}}$} & \multicolumn{2}{|c|}{$2^{\text {nd }}$} & \multicolumn{2}{|c|}{$3^{\text {rd }}$} & \multicolumn{2}{|c|}{$4^{\text {th }}$} & \multicolumn{2}{|c|}{$5^{\text {th }}$} \\
\hline & $\%$ Var. & $\%$ Acc. & $\%$ Var. & $\%$ Acc. & $\%$ Var. & $\%$ Acc. & $\%$ Var. & $\%$ Acc. & $\%$ Var. & $\%$ Acc. \\
\hline $\mathrm{MF}$ & 77.77 & 77.77 & 6.07 & 83.84 & 5.09 & 88.93 & 2.58 & 91.51 & 1.52 & 93.03 \\
\hline IAF & 81.48 & 81.48 & 7.01 & 88.48 & 3.52 & 92.00 & 1.86 & 93.86 & 1.29 & 95.15 \\
\hline $\mathrm{TF}$ & 75.46 & 75.46 & 8.00 & 83.46 & 5.54 & 89.01 & 2.75 & 91.76 & 1.63 & 93.39 \\
\hline SEF95 & 76.71 & 76.71 & 7.35 & 84.06 & 3.64 & 87.70 & 3.15 & 90.85 & 2.19 & 93.04 \\
\hline SE & 75.62 & 75.62 & 7.09 & 82.71 & 3.62 & 86.33 & 3.03 & 89.35 & 2.19 & 91.54 \\
\hline
\end{tabular}


Table 3. Sensitivity, specificity, accuracy, area under ROC curve (AUC) and $p$-values obtained with the first principal component for each parameter: mean frequency (MF), individual alpha frequency (IAF), transition frequency (TF), 95\% spectral edge frequency (SEF95) and spectral entropy (SE).

\begin{tabular}{cccccc}
\hline Parameters & Sensitivity $(\%)$ & Specificity $(\%)$ & Accuracy $(\%)$ & AUC & $p$-value \\
\hline MF & 85.00 & 85.71 & 85.37 & 0.912 & $<0.00001$ \\
IAF & 80.00 & 80.95 & 80.49 & 0.821 & $<0.00005$ \\
TF & 70.00 & 76.19 & 73.17 & 0.760 & 0.00190 \\
SEF95 & 65.00 & 66.67 & 65.85 & 0.698 & 0.01760 \\
SE & 90.00 & 76.19 & 82.93 & 0.888 & 0.00023 \\
\hline
\end{tabular}

Table 4. Classification results of the stepwise discriminant analyses.

\begin{tabular}{lcll}
\hline Parameter combinations & Sensitivity $(\%)$ & Specificity $(\%)$ & Accuracy $(\%)$ \\
\hline MF, SEF95, TF & $16 / 20(80.00)$ & $20 / 21(95.24)$ & $36 / 41(87.80)$ \\
TF, SEF95, SE & $15 / 20(75.00)$ & $21 / 21(100.00)$ & $36 / 41(87.80)$ \\
IAF, SEF95, SE & $15 / 20(75.00)$ & $21 / 21(100.00)$ & $36 / 41(87.80)$ \\
MF, IAF, SEF95, TF & $16 / 20(80.00)$ & $20 / 21(95.24)$ & $36 / 41(87.80)$ \\
MF, IAF, SEF95, SE & $15 / 20(75.00)$ & $21 / 21(100.00)$ & $36 / 41(87.80)$ \\
IAF, SEF95, TF, SE & $15 / 20(75.00)$ & $21 / 21(100.00)$ & $36 / 41(87.80)$ \\
\hline
\end{tabular}




\section{Conflict of interest statement}

There are no conflicts of interest that could inappropriately influence this research work. 